\title{
Comparative Cost per Response for 4 Clinical Endpoints with Tocilizumab Monotherapy vs Adalimumab Monotherapy in a Head-to-Head Randomized Double-Blind Superiority Trial (ADACTA) in Patients with Rheumatoid Arthritis
}

Jennie H. Best · Steven C. Vlad · Jinglan Pei

Received: November 7, 2019 / Published online: January 6, 2020

(C) The Author(s) 2020

\begin{abstract}
Introduction: The cost-effectiveness of different biologic therapies can be an important component in guiding treatment decisions for patients with rheumatoid arthritis (RA). The objective of this study was to compare drug and adverse event costs and cost per successful clinical response with tocilizumab (TCZ) monotherapy vs adalimumab (ADA) monotherapy in patients with RA in a phase 4 clinical trial.

Methods: Patients received either TCZ intravenously every 4 weeks or ADA subcutaneously every 2 weeks for 24 weeks. Drug and administration costs were based on wholesale acquisition costs and the Centers for Medicare and Medicaid, respectively. Outcomes included patient-level drug costs, cost of hospitalization
\end{abstract}

Enhanced Digital Features To view enhanced digital features for this article go to https://doi.org/10.6084/ m9.figshare.11353616.

J. H. Best $(\bowtie) \cdot$ J. Pei

Genentech, Inc., South San Francisco, CA, USA

e-mail: best.jennie@gene.com

S. C. Vlad

Department of Rheumatology, Tufts Medical

Center, Boston, MA, USA due to adverse events, and cost per response. Cost per response was calculated by dividing the mean drug plus administration cost by the proportion of patients achieving Disease Activity Score in 28 joints (DAS28) $<2.6$ (remission) or $20 \%, 50 \%$, or $70 \%$ improvement in response per the American College of Rheumatology (ACR20/50/70). Hospitalization costs were calculated using the daily hospital cost and number of hospital days.

Results: Among the 163 patients treated with TCZ and 162 patients treated with ADA, mean total drug and administration costs per patient over 24 weeks were $\$ 18,290.60$ and $\$ 25,623.10$, respectively. Mean drug and administration costs per each clinical response achieved were lower with TCZ than with ADA (DAS28 $<2.6$ : $\$ 45,868$ vs $\$ 244,174 ;$ ACR20: $\$ 28,127$ vs $\$ 51,887$; ACR50: $\$ 38,720$ vs $\$ 92,244$; ACR70: $\$ 56,253$ vs $\$ 143,136)$. The total hospital days were 32 days with TCZ and 43 days with ADA; mean hospital costs per patient were $\$ 484.50$ with TCZ and \$651.10 with ADA.

Conclusion: In this comparative assessment, the cost to achieve all 4 clinical endpoints was lower for patients receiving TCZ than for those receiving ADA.

Keywords: Cost per responder; Rheumatoid arthritis; Tocilizumab; Treatment costs 


\section{Key Summary Points}

\section{Why carry out this study?}

The cost-effectiveness of different biologic therapies can be an important component in guiding treatment decisions for patients with rheumatoid arthritis (RA)

This analysis compared drug and adverse event costs and cost per successful clinical response with tocilizumab (TCZ) monotherapy vs adalimumab (ADA) monotherapy in patients with RA in a phase 4 clinical trial (ADACTA)

\section{What was learned from this study?}

Using data from the ADACTA trial, this analysis found that the cost to achieve clinical response was lower in patients with RA who received TCZ monotherapy than in those who received ADA monotherapy

In addition, hospitalization costs were lower in patients who received TCZ than in those who received ADA

\section{INTRODUCTION}

Rheumatoid arthritis (RA) is a chronic autoimmune disease characterized by joint pain, swelling, and stiffness. RA is associated with substantial costs resulting from direct sources, including drug therapy and hospitalization-related expenses, and indirect sources such as reduced productivity $[1,2]$. One of the largest components of total RA costs is drug expenses, which have risen in response to the increased use and availability of biologic disease-modifying antirheumatic drugs (DMARDs) to treat RA $[2,3]$. Thus, comparisons of the economic value of different biologic therapies can be an important consideration in guiding treatment decisions for patients with RA.

Tocilizumab (TCZ) is a humanized monoclonal antibody that blocks the interleukin- 6 receptor and is approved for the treatment of patients with moderate to severe RA who have had an inadequate response to at least one DMARD [4]. TCZ has been shown to be safe and effective when administered as monotherapy [5-7] or in combination with methotrexate (MTX) $[6,8,9]$. The anti-tumor necrosis factor agent (aTNF) adalimumab (ADA) is a fully human monoclonal antibody that is approved for the treatment of patients with moderate to severe RA either alone or in combination with DMARDs [10] and recommended for use after an inadequate response to at least one DMARD $[11,12]$.

The efficacy of these two biologics was evaluated in the ADACTA trial, a 24-week randomized controlled phase 4 superiority study that compared TCZ monotherapy with ADA monotherapy in patients with RA who were intolerant of MTX or who were inappropriate for continued MTX treatment [7]. This study demonstrated that TCZ monotherapy was more effective than ADA monotherapy in reducing the signs and symptoms of RA over 24 weeks.

Few studies have compared the cost-effectiveness of TCZ and ADA in patients with RA. Furthermore, hospitalization costs are often excluded from analyses of the cost-effectiveness of DMARDs $[13,14]$. The objective of this study was to determine the cost per successful outcome in patients with RA who received TCZ or ADA using data from the ADACTA study.

\section{METHODS}

\section{Patient Population}

The study population for this analysis was the intent-to-treat population from ADACTA, a randomized, double-blind, multicenter superiority study that compared the efficacy of intravenously administered (IV) TCZ $8 \mathrm{mg} / \mathrm{kg}$ every 4 weeks $(n=163)$ with that of subcutaneously administered (SC) ADA $40 \mathrm{mg}$ every 2 weeks $(n=162)$ for 24 weeks in patients with active RA [7]. The inclusion criteria in the ADACTA trial have been described in detail [7]. Briefly, patients were aged 18 years or more, had severe RA for at least 6 months, were intolerant of 
MTX or were inappropriate candidates for continued MTX treatment, and had not received a prior biologic. Patients were required to have an erythrocyte sedimentation rate of greater than $28 \mathrm{~mm} / \mathrm{h}$ or C-reactive protein level of at least $1.0 \mathrm{mg} / \mathrm{dL}$ during screening and a Disease Activity Score in 28 joints (DAS28) greater than 5.1 , swollen joint count of at least 6 , and tender joint count of at least $8(66 / 68$ joint count) at baseline.

This retrospective analysis used data from the ADACTA study; the ADACTA study protocol was approved by an ethics committee or institutional review board at each participating center and was conducted in accordance with the Declaration of Helsinki. All patients provided written informed consent.

\section{Data Source}

Drug costs were obtained from AnalySource-First Databank [15] and were based on wholesale acquisition cost (WAC) drug prices (July 2018). Administration costs (IV TCZ only) were based on the Centers for Medicare and Medicaid 2018 Healthcare Common Procedure Coding System (HCPCS) code 96413. The daily cost of hospitalization (private and nonprivate) was based on the American Hospital Association 2015 report, updated to 2018 .

\section{Study Outcomes}

Mean patient-level drug costs and cost of hospitalization due to adverse events (AEs) were assessed at 24 weeks. For TCZ, drug costs were reported as $\$ 436.80$ per $80 \mathrm{mg}(4 \mathrm{~mL})$ vial based on weight at baseline (dosed at $10 \mathrm{~kg} / \mathrm{vial}$, rounded up to next higher vial), and administration costs were reported as $\$ 145.00$ for each infusion. For ADA, drug costs were reported as $\$ 2436.00$ per $40 \mathrm{mg}$, with no costs associated with administration. To estimate hospitalization costs for each biologic, the total cost was calculated as the number of hospital days per group multiplied by the daily cost of hospitalization. The daily cost of hospitalization (private and nonprivate) was based on the
American Hospital Association 2016 report, updated to 2018 using the Consumer Price Index for health care for 2017 (2.46\%) and 2018 $(2.4 \%): \quad(\$ 2338 \times 1.0246) \times 1.024=\$ 2453$. Cost per response was evaluated for each drug using DAS28 remission criteria (DAS28 $<2.6$ ) and $20 \%, 50 \%$, or $70 \%$ improvement in response per the American College of Rheumatology (ACR20/50/70). Cost per response was calculated by dividing the mean drug plus administration cost by the proportion of patients achieving DAS28 $<2.6$ (remission), ACR20, ACR50, or ACR70.

In a secondary analysis, the number needed to treat (NNT) was also calculated.

\section{RESULTS}

Among the 163 patients included in the TCZ group, the total drug and administration costs were $\$ 2,981,372$. For the 162 patients included in the ADA group, the total drug and administration costs were $\$ 4,150,944$. The mean drug and administration cost per patient was $\$ 18,290.60$ in the TCZ group and $\$ 25,623.10$ in the ADA group.

In the TCZ group, five patients were hospitalized for six AEs for a total of 32 days. The total hospitalization costs among patients treated with TCZ were estimated as $\$ 78,496$, for a mean cost per patient of $\$ 484.50$ (Table 1$)$. In the ADA group, five patients were hospitalized for eight AEs for a total of 43 days. The total hospitalization cost among ADA-treated patients was estimated as $\$ 105,479$, for a mean cost per patient of $\$ 651.10$.

In ADACTA, a significantly higher proportion of patients who received TCZ monotherapy achieved DAS28 $<2.6$, ACR20, ACR50, or ACR70 at 24 weeks than those who received ADA monotherapy (Fig. 1) [7]. Mean cost per response at 24 weeks was numerically higher in patients who received ADA than in those who received TCZ for DAS28 $<2.6(\$ 244,174$ vs $\$ 45,868)$, ACR20 ( $\$ 51,887$ vs $\$ 28,127)$, ACR50 $(\$ 92,244$ vs $\$ 38,720)$, and ACR70 (\$143,136 vs $\$ 56,253$ ) (Fig. 2).

The NNT analysis showed that there was an additional responder for every 3.4, 6.4, 
Table 1 Mean hospitalization costs over 24 weeks

\begin{tabular}{|c|c|c|}
\hline & $\begin{array}{l}\text { TCZ } \\
(n=163)\end{array}$ & $\begin{array}{l}\text { ADA } \\
(n=162)\end{array}$ \\
\hline No. of patients hospitalized & 5 & 5 \\
\hline No. of AEs requiring hospitalization & 6 & 8 \\
\hline Total hospital days & 32 & 43 \\
\hline Total costs of hospitalization, $\$^{\mathrm{a}}$ & $78,496.00$ & $105,479.00$ \\
\hline Mean cost per patient, $\$^{\mathrm{a}}$ & 484.50 & 651.10 \\
\hline
\end{tabular}

$A D A$ adalimumab, $A E$ adverse event, $T C Z$ tocilizumab

${ }^{a}$ Based on a hospitalization cost per day of $\$ 2453$

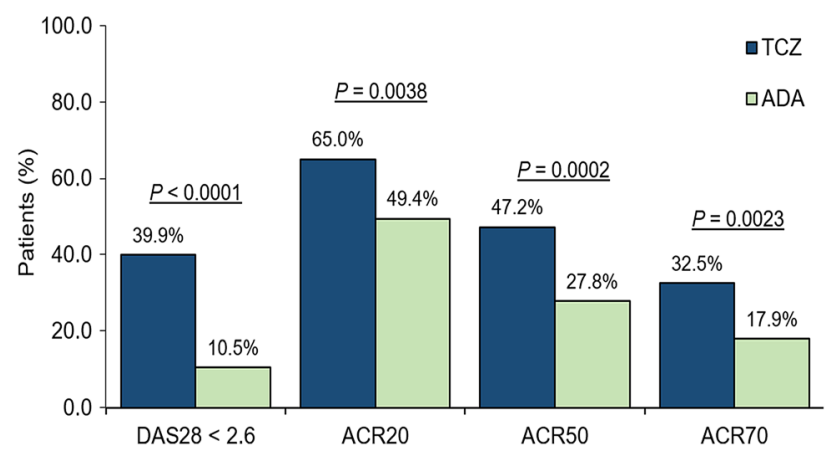

Fig. 1 Clinical response at 24 weeks in patients randomized to either TCZ or ADA [7]. ACR20/50/70 20\%, 50\%, or $70 \%$ improvement in response, respectively, per the
American College of Rheumatology, $A D A$ adalimumab, $D A S 28$ Disease Activity Score in 28 joints, $T C Z$ tocilizumab

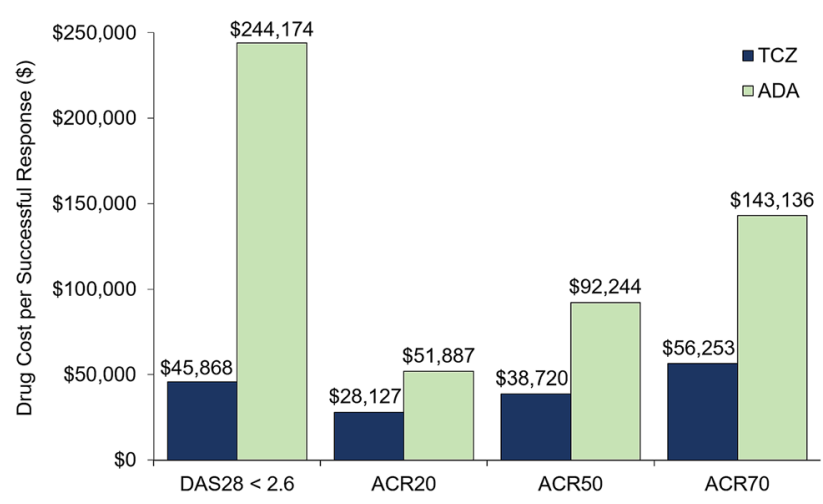

Fig. 2 Mean cost (drug + administration) per successful response achieved at 24 weeks. ACR20/50/70 20\%, 50\%, or $70 \%$ improvement in response, respectively, per the

5.2, and 6.8 patients treated with TCZ compared with patients treated with ADA for DAS28 < 2.6, ACR20, ACR50, or ACR70, respectively.
American College of Rheumatology, $A D A$ adalimumab, DAS28 Disease Activity Score in 28 joints, $T C Z$ tocilizumab

\section{DISCUSSION}

This analysis investigated the cost per response of TCZ monotherapy and ADA monotherapy in 
patients with RA from a US payer perspective. At 24 weeks of treatment, drug and administration costs and hospitalization costs were lower in patients receiving TCZ than in patients receiving $\mathrm{ADA}$. Correspondingly, the mean cost per clinical response for 4 different measures of efficacy was lower in patients receiving TCZ than in those receiving ADA.

Several studies evaluated the cost-effectiveness of TCZ and ADA in patients with RA. The Institute for Clinical and Economic Review reported that TCZ IV monotherapy was less costly and more effective than ADA [14]. In addition, a US-based assessment of annual treatment-related (drug plus administration) costs estimated that the cost per ACR20 responder with SC ADA was numerically higher than the cost per responder with SC TCZ when both biologics were administered as monotherapy $(\$ 86,096$ vs $\$ 62,690)$ [16]. Despite varied methodologies and patient populations and differences in TCZ use, these studies largely support our results and suggest that IV or SC TCZ monotherapy is a more cost-effective treatment than ADA monotherapy. The present findings are also aligned with a prior comparison of the cost-effectiveness of TCZ vs ADA using data from the ADACTA trial, in which a model-based analysis of lifetime cost-effectiveness showed that, despite the higher treatmentrelated costs (drug acquisition, administration, and monitoring) of TCZ monotherapy compared with ADA monotherapy $(\$ 15,636$ vs $\$ 13,553$ at 6 months), TCZ was projected to be more cost-effective than ADA, with an incremental cost-effectiveness ratio of $\$ 36,944$ per quality-adjusted life-year [13].

Although the concomitant use of conventional synthetic DMARDs (csDMARDs) is recommended with aTNFs (such as ADA) owing to their ability to reduce immunogenicity and increase efficacy $[12,17,18]$, real-world studies have shown that as many as $25-45 \%$ of patients use biologic DMARDs as monotherapy (no concomitant csDMARDs) [19-22]. The results of the present study, along with the proven efficacy and safety of both IV and SC TCZ, demonstrate the value of TCZ for patients who cannot tolerate or prefer not to use MTX. Future studies utilizing registry or claims data with longer follow-up of patients typically treated in routine clinical practice (i.e., multiple comorbidities) are warranted to confirm these results.

This study has some limitations. This analysis was based on wholesale acquisition cost prices and did not consider real-world treatment patterns or any contracted discounts or rebates provided by manufacturers. In addition, as our analysis was based on ADACTA data assessing clinical response rate at 24 weeks of treatment, this study did not account for longer duration of treatment or treatment with a different dosage and/or frequency of administration. This analysis was also limited to IV TCZ treatment and did not evaluate costs for SC TCZ treatment. However, comparable efficacy and safety between SC and IV TCZ have been shown in clinical trials [23-25], and comparable effectiveness with SC and IV TCZ has been shown in a real-world setting [23]. Finally, it is possible that hospitalization rates reported in clinical trials such as ADACTA may differ from those observed in routine clinical practice.

\section{CONCLUSIONS}

Using data from the ADACTA trial, this analysis found that the cost to achieve all 4 clinical responses was lower in patients with RA who received TCZ monotherapy than in those who received ADA monotherapy. In addition, hospitalization costs were lower in patients who received TCZ than in those who received ADA.

\section{ACKNOWLEDGEMENTS}

Funding. This work and the Rapid Service Fee were funded by Genentech, Inc. All authors had full access to all data in this study and take complete responsibility for the integrity of the data and accuracy of the data analysis.

Medical Writing Assistance. Support for third-party writing assistance, furnished by Nicola Gillespie, DVM, of Health Interactions, Inc, was provided by Genentech, Inc. 
Authorship. All named authors meet the International Committee of Medical Journal Editors (ICMJE) criteria for authorship for this article, take responsibility for the integrity of the work as a whole, and have given their approval for this version to be published.

Authorship Contributions. All authors were involved in drafting the manuscript, reviewing, and revising it critically for important intellectual content, and all authors read and approved the final version. All authors also contributed to the study design, analysis, and interpretation of data.

Disclosures. Jennie H. Best and Jinglan Pei are employees and shareholders of Genentech, Inc. Steven C. Vlad has received speakers bureau fees from AbbVie $(<\$ 10,000)$, consulting fees from Kiniksa, Horizon, AbbVie, and UCB $(<\$ 10,000)$, and research funding from Regeneron, Centrexion, AbbVie, UCB, GSK, Samumed, Galapagos, Eli Lilly, and Novartis.

Compliance with Ethics Guidelines. This retrospective analysis used data from the ADACTA study; the ADACTA study protocol was approved by an ethics committee or institutional review board at each participating center and was conducted in accordance with the Declaration of Helsinki. All patients provided written informed consent.

Data Availability. Qualified researchers may request access to individual patient-level data analyzed for this study through the clinical study data request platform (www. clinicalstudydatarequest.com). Further details on Roche's criteria for eligible studies are available here (https://clinicalstudydatarequest.com/ Study-Sponsors/Study-Sponsors-Roche.aspx).

For further details on Roche's Global Policy on the Sharing of Clinical Study Information and how to request access to related clinical study documents, see here (https://www.roche.com/ research_and_development/who_we_are_how_ we_work/clinical_trials/our_commitment_to_ data_sharing.htm).
Open Access. This article is distributed under the terms of the Creative Commons Attribution-NonCommercial 4.0 International License (http://creativecommons.org/licenses/ by-nc/4.0/), which permits any noncommercial use, distribution, and reproduction in any medium, provided you give appropriate credit to the original author(s) and the source, provide a link to the Creative Commons license, and indicate if changes were made.

\section{REFERENCES}

1. Kvien TK. Epidemiology and burden of illness of rheumatoid arthritis. Pharmacoeconomics. 2004;22(1):1-12.

2. Kobelt G, Woronoff A-S, Richard B, Peeters P, Sany J. Disease status, costs and quality of life of patients with rheumatoid arthritis in France: the ECO-PR Study. Joint Bone Spine. 2008;75(4):408-15.

3. Michaud K, Messer J, Choi HK, Wolfe F. Direct medical costs and their predictors in patients with rheumatoid arthritis: a three-year study of 7,527 patients. Arthritis Rheum. 2003;48(10):2750-62.

4. ACTEMRA Prescribing Information. Genentech, Inc. South San Francisco, CA. 2019.

5. Jones G, Sebba A, Gu J, et al. Comparison of tocilizumab monotherapy versus methotrexate monotherapy in patients with moderate to severe rheumatoid arthritis: the AMBITION study. Ann Rheum Dis. 2010;69(1):88-96.

6. Burmester GR, Rigby WF, van Vollenhoven RF, et al. Tocilizumab in early progressive rheumatoid arthritis: FUNCTION, a randomised controlled trial. Ann Rheum Dis. 2016;75(6):1081-91.

7. Gabay C, Emery P, van Vollenhoven R, et al. Tocilizumab monotherapy versus adalimumab monotherapy for treatment of rheumatoid arthritis (ADACTA): a randomised, double-blind, controlled phase 4 trial. Lancet. 2013;381(9877):1541-50.

8. Genovese MC, McKay JD, Nasonov EL, et al. Interleukin-6 receptor inhibition with tocilizumab reduces disease activity in rheumatoid arthritis with inadequate response to disease-modifying antirheumatic drugs: the tocilizumab in combination with traditional disease-modifying antirheumatic drug therapy study. Arthritis Rheum. 2008;58(10): 2968-80. 
9. Smolen JS, Beaulieu A, Rubbert-Roth A, et al. Effect of interleukin-6 receptor inhibition with tocilizumab in patients with rheumatoid arthritis (OPTION study): a double-blind, placebo-controlled, randomised trial. Lancet. 2008;371(9617):987-97.

10. HUMIRA Prescribing Information. AbbVie Inc. North Chicago, IL: 2014.

11. Singh JA, Saag KG, Bridges SL Jr, et al. 2015 American College of Rheumatology guideline for the treatment of rheumatoid arthritis. Arthritis Care Res (Hoboken). 2016;68(1):1-25.

12. Smolen JS, Landewe R, Bijlsma J, et al. EULAR recommendations for the management of rheumatoid arthritis with synthetic and biological diseasemodifying antirheumatic drugs: 2016 update. Ann Rheum Dis. 2017;76(6):960-77.

13. Carlson JJ, Ogale S, Dejonckheere F, Sullivan SD. Economic evaluation of tocilizumab monotherapy compared to adalimumab monotherapy in the treatment of severe active rheumatoid arthritis. Value Health. 2015;18(2):173-9.

14. Institute for Clinical and Economic Review. Targeted immune modulators for rheumatoid arthritis: effectiveness and value. 2017; https://icer-review. org/material/ra-final-report/. Accessed Apr 7, 2019.

15. First Databank, AnalySource ${ }^{\circledR}$ Online. Wholesale Acquisition Cost (WAC). San Francisco, CA. 2017; http://www.fdbhealth.com/fdb-medknowledgedrug-pricing. Accessed Jul 1, 2018.

16. Claxton L, Jenks M, Taylor M, et al. An economic evaluation of tofacitinib treatment in rheumatoid arthritis: modeling the cost of treatment strategies in the United States. J Manag Care Spec Pharm. 2016;22(9):1088-102.

17. Buckley F, Finckh A, Huizinga TW, Dejonckheere F, Jansen JP. Comparative efficacy of novel DMARDs as monotherapy and in combination with methotrexate in rheumatoid arthritis patients with inadequate response to conventional DMARDs: a network meta-analysis. J Manag Care Spec Pharm. 2015;21(5):409-23.

18. Breedveld FC, Weisman MH, Kavanaugh AF, et al. The PREMIER study: a multicenter, randomized, double-blind clinical trial of combination therapy with adalimumab plus methotrexate versus methotrexate alone or adalimumab alone in patients with early, aggressive rheumatoid arthritis who had not had previous methotrexate treatment. Arthritis Rheum. 2006;54(1):26-37.

19. Catay E, Bravo M, Rosa J, Soriano ER. Prevalence of biologics monotherapy in a cohort of patients with rheumatoid arthritis in daily clinical practice. BMC Musculoskelet Disord. 2016;17:110.

20. Choquette D, Arundine M, Thomas OC. Large discrepancy between expected and observed ratios of biologic treated rheumatoid arthritis patients also compliant on DMARDs. Ann Rheum Dis. 2011;70(Suppl 3):197.

21. Choy E, Aletaha D, Behrens F, et al. Monotherapy with biologic disease-modifying anti-rheumatic drugs in rheumatoid arthritis. Rheumatology (Oxford). 2017;56(5):689-97.

22. Pappas DA, Reed GW, Saunders K, et al. Characteristics associated with biologic monotherapy use in biologic-naive patients with rheumatoid arthritis in a US registry population. Rheumatol Ther. 2015;2:85-95.

23. Lauper K, Nordström DC, Pavelka K, et al. Comparative effectiveness of tocilizumab versus TNF inhibitors as monotherapy or in combination with conventional synthetic disease-modifying antirheumatic drugs in patients with rheumatoid arthritis after the use of at least one biologic diseasemodifying antirheumatic drug: analyses from the pan-European TOCERRA register collaboration. Ann Rheum Dis. 2018;77(9):1276-82.

24. Ogata A, Tanimura K, Sugimoto T, et al. Phase III study of the efficacy and safety of subcutaneous versus intravenous tocilizumab monotherapy in patients with rheumatoid arthritis. Arthritis Care Res (Hoboken). 2014;66(3):344-54.

25. Burmester GR, Rubbert-Roth A, Cantagrel A, et al. A randomised, double-blind, parallel-group study of the safety and efficacy of subcutaneous tocilizumab versus intravenous tocilizumab in combination with traditional disease-modifying antirheumatic drugs in patients with moderate to severe rheumatoid arthritis (SUMMACTA study). Ann Rheum Dis. 2014;73(1):69-74. 